\title{
Biomaterial antifúngico para reducir las infecciones causadas por Candida albicans en pacientes edéntulos
}

\author{
Laura S. Acosta-Torres, ${ }^{1 *}$ Juan C. Flores-Arriaga, ${ }^{2}$ Paloma N. Serrano-Díaz, ${ }^{1}$ Itzel A. González-García, ${ }^{1}$ \\ Juan C. Viveros-García, ${ }^{3}$ Ma. del Carmen Villanueva-Vilchis, ${ }^{4}$ Francisco G. Villanueva-Sánchez, ${ }^{5}$ \\ René García-Contreras ${ }^{1}$ y Ma. Concepción Arenas-Arrocena ${ }^{1}$ \\ ${ }^{1}$ Área Nanoestructuras y Biomateriales, Laboratorio de Investigación Interdisciplinaria, Universidad Nacional Autónoma de México, Escuela Nacional \\ de Estudios Superiores Unidad León, Guanajuato; 'Universidad Autónoma de San Luis Potosí, Facultad de Estomatología, Laboratorio de Ciencias \\ Básicas, San Luis Potosí; ${ }^{3}$ Área Geriatría, Licenciatura en Fisioterapia, Universidad Nacional Autónoma de México, Escuela Nacional de Estudios \\ Superiores Unidad León, Guanajuato; ' Área de Salud Pública Bucal, Licenciatura en Odontología, Universidad Nacional Autónoma de México, \\ Escuela Nacional de Estudios Superiores Unidad León, Guanajuato; 5 Área Patología Oral y Maxilofacial, Laboratorio de Investigación Interdisciplinaria, \\ Universidad Nacional Autónoma de México, Escuela Nacional de Estudios Superiores Unidad León, Guanajuato. México
}

\section{Resumen}

Introducción: Las resinas acrílicas de polimetilmetacrilato (PMMA) son utilizadas para elaborar dentaduras para pacientes edéntulos. Objetivo: Conocer la prevalencia de las especies de Candida en pacientes con y sin prótesis removibles de una clínica de odontología en León, Guanajuato; así como valorar el efecto antifúngico y el comportamiento biológico de un PMMA experimental con nanopartículas de plata para su posible aplicación en prótesis. Métodos: Para identificar las especies de Candida se obtuvieron muestras para frotis de la mucosa palatina de 140 pacientes con edad $\geq 60$ años. El PMMA experimental con nanopartículas de plata fue colocado en cultivos de Candida albicans, los cuales fueron teñidos con el kit Live/ Dead® para su análisis bajo microscopia confocal; posteriormente, se implantó en ratas Wistar para conocer su comportamiento en los tejidos circundantes. Resultados: Candida albicans fue la especie más prevalente en los pacientes valorados, seguida de Candida tropicalis y Candida krusei. La resina acrílica con nanopartículas de plata disminuyó significativamente la presencia de Candida albicans. En el modelo animal se encontró reacción inflamatoria discreta y controlada, lo cual indicó la biocompatibilidad de la resina acrílica utilizada. Conclusiones: Es posible utilizar el material nanoestructurado con efecto antifúngico para promover la reducción de infecciones orales por Candida en pacientes edéntulos.

PALABRAS CLAVE: Nanopartículas de plata. Resina acrílica. Antifúngico. Biocompatibilidad en modelo animal.

\section{Antifungal biomaterial for reducing infections caused by Candida albicans in edentulous patients}

\section{Abstract}

Introduction: Polymethylmethacrylate (PMMA) acrylic resins are used to make dentures for edentulous patients. Objective: To find out the prevalence of Candida species in patients with and without removable prostheses from a dental clinic in León, Guanajuato, as well as to assess the antifungal effect and biological behavior of an experimental PMMA with silver nanoparticles for its possible application in prostheses. Method: To identify Candida species, smear samples were obtained from the palatal mucosa of 140 patients aged $\geq 60$ years. The experimental PMMA with silver nanoparticles was placed in Candida albicans cultures, which were stained with the Live/Dead ${ }^{\otimes}$ kit for analysis under confocal microscopy; subsequently, it was implanted in

Correspondencia:

*Laura S. Acosta-Torres

E-mail: lacosta@enes.unam.mx
Fecha de recepción: 09-12-2020

Fecha de aceptación: 21-01-2021

DOI: 10.24875/GMM.20000915
Gac Med Mex. 2021;157:437-442

Disponible en PubMed

www.gacetamedicademexico.com

0016-3813/@ 2021 Academia Nacional de Medicina de México, A.C. Publicado por Permanyer. Este es un artículo open access bajo la licencia CC BY-NC-ND (http://creativecommons.org/licenses/by-nc-nd/4.0/). 
Wistar rats in order to know its behavior in the surrounding tissues. Results: Candida albicans was the most prevalent species in the evaluated patients, followed by Candida tropicalis and Candida krusei. The acrylic resin with silver nanoparticles significantly decreased the presence of Candida albicans. In the animal model, a discrete and controlled inflammatory reaction was found, which indicated biocompatibility of the acrylic resin that was used. Conclusions: It is possible for the nanostructured material with antifungal effect to be used in order to promote the reduction of oral Candida infections in edentulous patients.

KEY WORDS: Silver nanoparticles. Acrylic resin. Antifungal. Biocompatibility in animal model.

\section{Introducción}

De acuerdo con la Encuesta Nacional de Salud y Nutrición de 2012, en México hay 8874 personas de 60 años o más, lo que significa $9.2 \%$ de la población estimada del país; mientras que para 2050 se estima que los adultos mayores conformarán cerca de $28.0 \%$ de la población. ${ }^{1}$ Respecto a la salud bucal de los pacientes, el edentulismo es la pérdida total de todos los dientes naturales que causa dificultades de la masticación y provoca deficiencias nutricionales, aislamiento social y depresión. ${ }^{2}$ El edentulismo provoca que los pacientes requieran el uso de prótesis, lo que los predispone a infecciones como estomatitis subprotésica por la colonización de Candida albicans, la cual en México tiene una prevalencia de $65 \% .^{3}$ Los microorganismos del género Candida son un grupo de levaduras con comportamiento oportunista que forman parte del ecosistema de la mucosa bucal; ${ }^{4}$ Candida albicans es la especie más común.

Por otra parte, el material con el que se confeccionan las prótesis totales 0 dentaduras es la resina acrílica de polimetil metacrilato (PMMA), porque presenta propiedades fisicoquímicas deseables, pero requiere higiene constante para evitar la acumulación de microorganismos. ${ }^{5}$ Además, existen múltiples factores predisponentes de candidiasis como reducción del flujo salival, uso de corticosteroides, prótesis bucales desajustadas, malnutrición, desórdenes endocrinos e inmunitarios, entre otros. ${ }^{6}$

Actualmente no existe un biomaterial comercial para dentaduras con efecto antimicrobiano; $\sin$ embargo, en odontología el uso de nanopartículas es una tecnología de frontera que puede modificar sustancialmente las propiedades físicas, químicas y biológicas de los materiales, por lo que el propósito de la presente investigación fue conocer las especies de Candida más prevalentes en pacientes portadores de prótesis mexicanos, así como evaluar el efecto antifúngico contra Candida albicans de una resina acrílica experimental con nanopartículas de plata para aplicación en dentaduras y la biocompatibilidad en un modelo animal.

\section{Métodos}

Se llevó a cabo un estudio transversal en una primera fase para determinar las características sociodemográficas de los pacientes y la prevalencia de especies de Cándida Se eligieron por conveniencia 140 pacientes de 60 años y más que acudieron a la Clínica de Odontología de la Escuela Nacional de Estudios Superiores Unidad León, Universidad Nacional Autónoma de México, durante 2019. A todos se les realizó historial clínica y se recolectó información sobre variables sociodemográficas como edad, sexo, estado civil y lugar de origen. También se registró si los pacientes eran usuarios o no de prótesis dentales.

\section{Toma de muestras para identificación de Candida}

Todos los pacientes firmaron el consentimiento informado antes de iniciar la toma de muestra de la mucosa. La obtención de muestras de la mucosa palatina se efectuó bajo condiciones de asepsia con kit Stuart ${ }^{\circledR}$ (Thermo Fisher Scientific, España) para colección y transporte de muestras biológicas. Cada tubo se rotuló y selló para su transportación al laboratorio. Las muestras fueron sembradas en cajas de petri con CHROMagar Candida, para lo cual se empleó el método de estriado; posteriormente se incubaron a $35 \pm 2{ }^{\circ} \mathrm{C}$ durante 48 horas. Se realizó el registro de resultados de acuerdo con la coloración de cada muestra, con base en las especificaciones del medio del contraste: colonias pintadas de verde, Candida albicans; colonias en rosa, Candida krusei; colonias en azul, Candida tropicalis. Se reportan los resultados de pacientes con y sin prótesis que presentaron o no crecimiento de las diferentes especies de Candida, conforme al análisis estadístico de $\chi^{2}$. 
Tabla 1. Total de pacientes portadores y no portadores de prótesis dentales que mostraron Candida albicans, tropicalis o krusei

\begin{tabular}{|c|c|c|c|c|}
\hline \multirow[t]{2}{*}{ Portación o no de prótesis } & \multicolumn{4}{|c|}{ Pacientes con Candida (\%) } \\
\hline & Total $(n)$ & albicans & tropicalis & krusei \\
\hline Pacientes portadores de prótesis parciales o totales & 48 & 35.4 & 16.7 & 31.3 \\
\hline \multirow[t]{2}{*}{ Pacientes no portadores de prótesis } & 92 & 29.7 & 16.7 & 12.2 \\
\hline & & $\begin{aligned} \chi^{2} & =0.480 \\
p & =0.489\end{aligned}$ & $\begin{aligned} \chi^{2} & =0.018, \\
p & =0.892\end{aligned}$ & $\begin{aligned} \chi^{2} & =7.412 \\
p & =0.006\end{aligned}$ \\
\hline
\end{tabular}

\section{Resina acrílica experimental y nanopartículas de plata}

Se utilizó una resina acrílica de PMMA sintetizada en el Laboratorio de Investigación Interdisciplinaria donde se llevó a cabo el estudio. El PMMA es un acrílico rosa formado de partículas esféricas de 10 a $30 \mu \mathrm{m}$ desarrollado por Acosta Torres et al., cuya síntesis y caracterización se publicó en 2014. ${ }^{7}$ Las nanopartículas de plata (AgNP) fueron sintetizadas en el mismo laboratorio y por el mismo grupo de investigación, cuya formulación se publicó en $2011 .{ }^{8}$ Estas nanopartículas tienen morfología semiesférica de 20 a $50 \mathrm{~nm}$. El PMMA con o sin nanopartículas de plata ha sido previamente evaluado en cultivos con fibroblastos gingivales humanos a las 24 y 48 horas, sin que se hayan reportado efectos citotóxicos ni diferencias significativas con el grupo control. ${ }^{7}$

\section{Elaboración de muestras de PMMA y PMMA-AgNP}

Se fabricaron discos de resina acrílica de PMMA y PMMA-AgNP $(0.2 \mu \mathrm{g} / \mathrm{mL}$ de AgNP), de $10 \mathrm{~mm}$ de diámetro $\times 2 \mathrm{~mm}$ de espesor ${ }^{7}$ para realizar las pruebas de efecto antifúngico y biocompatibilidad en un modelo animal $(n=10)$. Las muestras fueron lavadas y esterilizadas en luz ultravioleta durante 10 minutos.

\section{Evaluación del efecto antifúngico}

Biopelículas de Candida albicans (ATCC 90026) fueron depositadas sobre discos de PMMA y PMMAAgNP y observadas mediante microscopia confocal de barrido láser (Leica SP5®). Las muestras de acrílico se tiñeron durante 15 minutos antes de la obtención de imágenes con el colorante Live/dead BacLight $^{\mathrm{TM}}$ preparado con $1 \mathrm{~mL}$ de agua destilada estéril, $1 \mathrm{~mL}$ de colorante de ácido nucleico fluorescente verde SYTO $^{\text {TM }} 9,1 \mathrm{~mL}$ de fluorescente rojo tinción con ácido nucleico y yoduro de propidio.

\section{Biocompatibilidad en un modelo animal}

Mediante procedimiento quirúrgico se realizó el implante de discos de PMMA y PMMA-AgNP en el dorso de 30 ratas Wistar, para valorar su efecto a los 15 y 45 días $(n=15)$. Como grupo control se utilizaron 15 ratas Wistar a las que no se les implantó ningún disco. Transcurrido cada periodo de tiempo, se sacrificó a los animales y se obtuvieron muestras para biopsia de los tejidos circundantes de la zona del implante de los discos acrílicos, de acuerdo con la ISO 10993-6. ${ }^{9}$ Se prepararon las muestras para su procesamiento histológico, se montaron en bloques de parafina, se realizaron los cortes con micrótomo y la tinción con hematoxilina-eosina, para la observación con microscopio óptico. ${ }^{10}$

\section{Consideraciones éticas}

El protocolo para la toma de muestras del paladar de los pacientes fue aprobado por la Comisión de Ética en Investigación de la Escuela Nacional de Estudios Superiores Unidad León (CE_17/009); se obtuvo la participación voluntaria de los pacientes. El protocolo para el ensayo en modelo animal fue aprobado por la Comisión de Ética de la misma escuela (CEl.19_033_S8E).

\section{Resultados}

De los 140 pacientes valorados, $63.6 \%$ correspondió al sexo femenino. La edad media fue de 69 años (60 a 92 años). La distribución por estado civil mostró que $66.4 \%$ fue casado y $33.6 \%$, soltero. Por el estado de origen, $66.4 \%$ de los pacientes nació en Guanajuato y $33.63 \%$ en otro estado de la República. 
Tabla 2. Descripción del análisis histopatológico de las muestras obtenidas de los animales a los 15 y 45 días de exposición a PMMA y PMMA-AgNP

\begin{tabular}{|c|c|c|c|c|c|c|}
\hline \multirow[t]{2}{*}{ Característica } & \multicolumn{3}{|c|}{15 días } & \multicolumn{3}{|c|}{45 días } \\
\hline & Control & PMMA & PMMA-AgNP & Control & PMMA & PMMA-AgNP \\
\hline Tipo de inflamación & Ausente & 80 \% crónica & $60 \%$ mixta & Ausente & $100 \%$ mixta & $90 \%$ mixta \\
\hline Severidad & $\begin{array}{l}\text { Células } \\
\text { inflamatorias no } \\
\text { detectadas }\end{array}$ & $\begin{array}{l}40 \% \text { moderada, } \\
40 \% \text { leve }\end{array}$ & $\begin{array}{l}60 \% \text { moderada, } \\
20 \% \text { severa }\end{array}$ & $\begin{array}{l}\text { Células inflamatorias } \\
\text { no detectadas }\end{array}$ & $60 \%$ leve & $50 \%$ leve \\
\hline Extensión & $\begin{array}{l}\text { Células } \\
\text { inflamatorias no } \\
\text { detectadas }\end{array}$ & $\begin{array}{l}90 \% \text { limitada a la } \\
\text { cápsula }\end{array}$ & $\begin{array}{l}90 \text { \% limitada a } \\
\text { la cápsula }\end{array}$ & $\begin{array}{l}\text { Células inflamatorias } \\
\text { no detectadas }\end{array}$ & $\begin{array}{l}100 \text { \% limitada } \\
\text { a la cápsula }\end{array}$ & $\begin{array}{l}100 \text { \% limitada } \\
\text { a la cápsula }\end{array}$ \\
\hline $\begin{array}{l}\text { Reacción a cuerpo } \\
\text { extraño }\end{array}$ & Ausente & $70 \%$ presente & $80 \%$ presente & Ausente & $100 \%$ ausente & $90 \%$ ausente \\
\hline
\end{tabular}

A

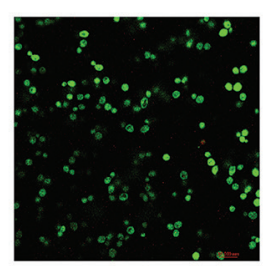

B

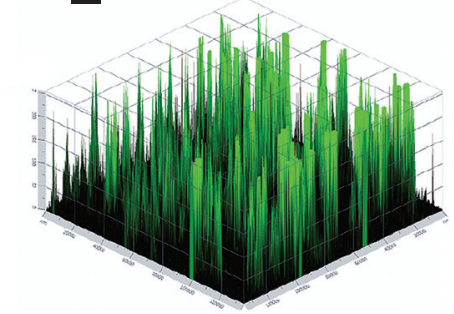

C

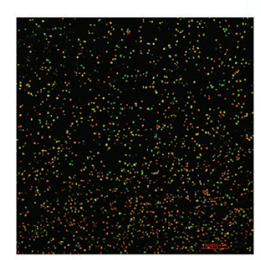

D

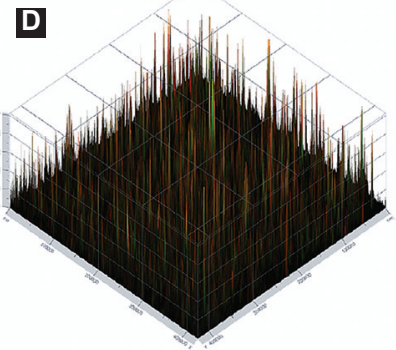

Figura 1. Microscopia confocal. A y B) Células de Candida albicans vivas (verde) sobre un sustrato de PMMA. C y D) células de Candida albicans muertas (rojo) sobre un sustrato de PMMA-AgNP, que indica daño por efecto de las nanopartículas de plata.

Se encontraron 48 pacientes portadores de prótesis, 21 con prótesis parcial y 27 con prótesis total (dentadura). Candida albicans se encontró en $65.1 \%$ de los pacientes, por lo cual constituyó la especie más prevalente. De acuerdo con los datos mostrados en la Tabla 1, se infirió que no existieron diferencias estadísticas significativas entre los pacientes positivos a Candida albicans si portaban o no prótesis. El mismo comportamiento se presentó en los pacientes portadores y no portadores de prótesis que resultaron positivos a Candida tropicalis. Sin embargo, se encontró diferencia significativa en los pacientes positivos a Candida krusei cuando usaban o no una prótesis dental, lo que mostró que el uso de prótesis favorece la proliferación de este microorganismo.

\section{Evaluación del efecto antifúngico}

Las imágenes de microscopia confocal muestran las superficies de los discos de PMMA y PMMA-AgNP en 2.5 D. En el grupo con PMMA se observaron células de Candida albicans en verde, que indica su viabilidad (Figuras $1 \mathrm{~A}$ y $1 \mathrm{~B}$ ). El grupo con PMMA-AgNP muestra fluorocromos verdes y rojos; el color rojo es indicativo de células muertas de Candida albicans (Figuras 1C y 1D).

\section{Evaluación histológica de PMMA y PMMA- AgNP en un modelo animal}

Los cortes histológicos evaluados evidencian la presencia de una pseudocápsula asociadas al nicho del material implantado; se observó una reacción inflamatoria de predominio linfoplasmocitario, discreta, controlada y limitada a la cápsula; así como células gigantes multinucleadas aisladas, lo que denota la biocompatibilidad del material utilizado.

La Figura 2 muestra las imágenes microscópicas de las células de infiltrado inflamatorio crónico y las células gigantes como prueba de la reacción a cuerpo extraño. Después de analizar el comportamiento celular de las muestras animales (Tabla 2), es posible decir que después de 45 días se observó $100 \%$ de inflamación mixta de severidad leve y que en $90 \%$ estaban ausentes las reacciones a cuerpo extraño. Por lo anterior, el material desarrollado se considera 

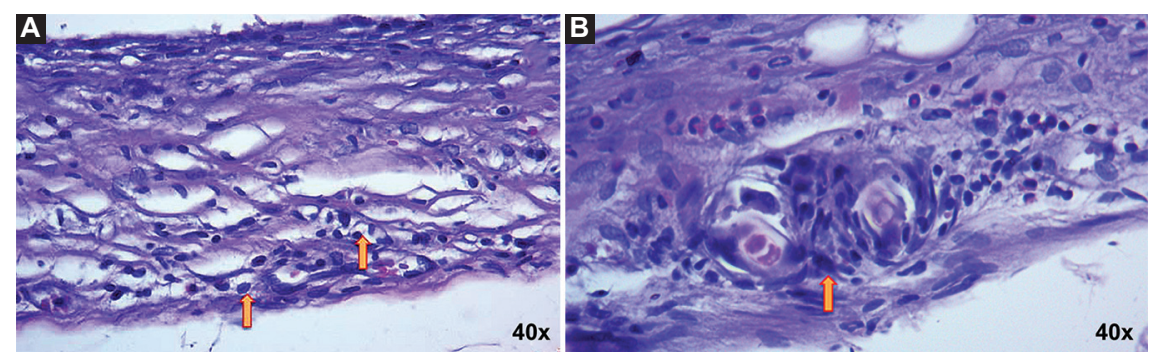

Figura 2. Análisis microscópico de las muestras histológicas tomadas de las ratas Wistar después de haber sido expuestas a la resina acrílica experimental. A) El tejido en contacto con PMMA muestra infiltrado inflamatorio crónico de predominio linfoplasmocitario (flechas). B) El tejido en contacto con PMMA-AgNP muestra reacción a cuerpo extraño, células gigantes aisladas (flechas). PMMA = polimetil metacrilato, $P M M A-A g N P=$ polimetil metacrilato con nanopartículas de plata.

biocompatible al ser evaluado en un modelo animal y viable para aplicación en la siguiente etapa de valoración clínica.

\section{Discusión}

La población mexicana que acude a las clínicas universitarias presenta alta prevalencia $(65.1 \%)$ de Candida albicans. En relación con el uso de prótesis, Bianchi CM et al., en 2016, realizaron un estudio en el que aislaron diferentes especies de Candida; encontraron que las personas de edad avanzada con prótesis removibles presentaron una probabilidad 4.4 veces mayor de aislamiento de Candida que las personas que no las usaban (IC $95 \%=1.65-11.4$ )..$^{11} \mathrm{En}$ contraste con el presente estudio, únicamente comprobamos la relación con el uso de prótesis con Candida krusei, probablemente debido a que solo 48 personas de la muestra total refirió usar prótesis.

El acrílico desarrollado con nanopartículas de plata cuenta con efecto antifúngico que inhibe Candida, lo que caodyuvará en el tratamiento cuando el paciente presente estomatitis subprotésica y posiblemente no tenga necesidad de tomar un tratamiento farmacológico adicional. En otros estudios, las nanopartículas de plata también han mostrado efecto antimicrobiano, sin ser tóxicas cuando tienen un tamaño de 20 a $50 \mathrm{~nm}$, y constituyen una posible alternativa al uso de antibióticos sin causar resistencia microbiana. ${ }^{12}$ Los reactivos utilizados para sintetizar las nanopartículas de plata son de bajo costo, por lo que esta opción podría estar al alcance de todos los pacientes. La resina acrílica propuesta es de fácil manipulación y disponible para los odontólogos y técnicos dentales que elaboran dentaduras, ya que el material puede reducir de forma directa la colonización microbiana y servir como un agente para prevenir las infecciones orales en adultos mayores.

\section{Conclusiones}

Se desarrolló una resina acrílica para dentaduras, a base de micropartículas de PMMA adicionado con nanopartículas de plata, como propuesta para los pacientes edéntulos que presenten infecciones por Candida. EI PMMA con nanopartículas de plata tiene efecto antifúngico en la inhibición de Candida albicans. Las pruebas previas in vitro del PMMA y PMMAAgNP indican que el material es no citotóxico, por lo que se consideró realizar un estudio in vivo no invasivo colocando la resina acrílica experimental en un modelo animal, lo que resultó biocompatible al evaluar el efecto en los tejidos vivos, por lo que se considerará proponer el producto para valoración clínica en pacientes.

\section{Agradecimientos}

Los autores agradecen a la Dirección General de Asuntos del Personal Académico de la Universidad Nacional Autónoma de México, por la beca posdoctoral del doctor Juan Carlos Flores Arriaga. Así como a la maestra Lourdes Palma Tirado, por el análisis microscópico electrónico de transmisión; a la doctora Genoveva Hernández Padrón, por el análisis de transformación infrarroja de Fourier; y a la doctora Marina Vega González, por el análisis microscópico electrónico de barrido.

\section{Conflicto de intereses}

Los autores declaran no tener conflicto de intereses alguno. 


\section{Financiamiento}

Los recursos para la presente investigación fueron obtenidos parcialmente de los siguientes proyectos PAPIIT-IN225516 y PAPIIT-IN115018.

\section{Responsabilidades éticas}

Protección de personas y animales. Los autores declaran que los procedimientos seguidos se conformaron a las normas éticas del comité de experimentación humana responsable y de acuerdo con la Asociación Médica Mundial y la Declaración de Helsinki.

Confidencialidad de los datos. Los autores declaran que siguieron los protocolos de su centro de trabajo sobre la publicación de datos de pacientes.

Derecho a la privacidad y consentimiento informado. Los autores obtuvieron el consentimiento informado de los pacientes o sujetos referidos en el artículo. Este documento obra en poder de la autora de correspondencia.

\section{Bibliografía}

1. Encuesta Nacional de Salud y Nutrición. Resultados Nacionales. México: Instituto Nacional de Salud Pública; 2012.

2. Gil-Montoya JA, Ferreira-de Mello AL, Barrios R, González-Moles MA, Bravo M. Oral health in the elderly patient and its impact on general well-being: a nonsystematic review. Clin Interv Aging. 2015;10:461-467.

3. Patil S, Rao RS, Majumdar B, Anil S. Clinical appearance of oral candida infection and therapeutic strategies. Front Microbiol. 2015;17:1391.

4. De la Rosa-García E, Miramontes-Zapata M, Sánchez-Vargas LO, Mondragón-Padilla A. Colonización e infección bucal por Candida sp. en pacientes diabéticos y no diabéticos con enfermedad renal crónica en diálisis. Nefrologia (Madr). 2013;33:764-770.

5. Pattanaik S, Bvj V, Pattanaik B, Sahu S, Lodam S. Denture stomatitis: a literature review. J Indian Acad Oral Med Radiol. 2010;22:136-140.

6. Darwazeh, AMG, Darwazeh TA. What makes. Oral candidiasis recurrent infection? A clinical view. J Mycol. 2014.

7. Acosta-Torres LS, Arenas-Arrocena MC, Núñez-Anita RE, Barceló-Santana FH, Álvarez-Gayosso C, Palacios-Alquisira J, et al. Nanopigmented acrylic resin cured indistinctively by water bath or microwave energy for dentures. J Nanomat. 2014;2014:3-10.

8. Acosta-Torres LS, Mendieta I, Núñez-Anita RE, Cajero-Juárez M, Castaño VM. Cytocompatible antifungal acrylic resin containing silver nanoparticles for dentures. Int J Nanomedicine. 2012;7:4777-4786.

9. Biological evaluation of medical devices - Part 6: tests for local effects after implantation [Internet]. Suiza: International Organization for Standarization; 2016

10. Taha NA, Safadi RA, Alwedaie MS. Biocompatibility evaluation of endosequence root repair paste in the connective tissue of rats. J Endod. 2016;42:1523-1528.

11. De Calvalho-Bianchi CMP, Aparecido-Bianchi H, Tadano T, Rodrigues-de Paula C, Hoffmann-Santos HD, Pereira-Leite D, Hahn RC. Factors related to oral candidiasis in elderly users and non-users of removable dental prostheses. Rev Inst Med Trop Sao Paulo. 2106;58:17.

12. Marambio-Jones $C$, Hoek EMV. A review of the antibacterial effects of silver nanomaterials and potential implications for human health and the environment. J Nanopart Res. 2010;12:1531-1551. 